\title{
OBESIDAD INFANTIL: UNA ENFERMEDAD MULTIDIMENSIONAL
}

\author{
CHILDHOOD OBESITY: A MULTIDIMENSIONAL DISEASE
}

La obesidad es una enfermedad prevalente en Argentina, aún desde edades pediátricas, con una tendencia creciente a lo largo de los últimos años en todos los grupos etarios y sociales, particularmente en aquellos en situación de mayor vulnerabilidad social. La Segunda Encuesta Nacional de Nutrición y Salud (2019) muestra un 41,1\% de exceso de peso en niños, niñas y adolescentes de 5 a 17 años, y de 13,6\% en menores de 5 años'.

La obesidad pone en riesgo la posibilidad real de niños, niñas y adolescentes de gozar plenamente de sus derechos. Si bien el derecho a la salud y a la alimentación son los más claramente afectados, la creciente prevalencia del sobrepeso compromete también el ejercicio de otros derechos, como el derecho a la educación, a la información, a la no discriminación, al juego y al esparcimiento y, más ampliamente, a la vida, a la supervivencia y al desarrollo pleno (UNICEF 2019) ${ }^{2}$. La obesidad pediátrica incrementa el riesgo de obesidad y de sus comorbilidades en la adultez.

Son múltiples los factores que intervienen e interactúan en su desarrollo: genéticos, epigenéti$\cos ^{3}$, ambientales intra y extrauterinos, socioeconómicos y familiares. El reconocimiento de estos factores es esencial para el establecimiento de estrategias de prevención. El estudio de Hirschler y col., publicado en esta edición, muestra la asociación entre peso de nacimiento y obesidad en niños de edad escolar. Si bien el peso es una medida cruda de la trayectoria de crecimiento intrauterino que no permite determinar per se la composición corporal del recién nacido, es muy influido por las condiciones de salud materna, antes y durante la gestación. La obesidad materna previa o durante el embarazo tiene una fuerte asociación con el alto peso de nacimiento y adiposidad, y con la obesidad en posterior ${ }^{4}$. Las intervenciones sobre la mujer dirigidas a un estilo de vida saludable, con una alimentación equilibrada e incremento de la actividad física antes y durante el embarazo para controlar la ganancia de peso, podrían reducir el riesgo del alto peso al nacer ${ }^{5}$. Además, las intervenciones preventivas dirigidas particularmente a esos niños con peso de nacimiento elevado podrían desviar sus trayectorias de crecimiento y prevenir su progresión a la obesidad. Promover la lactancia materna, la introducción oportuna de la alimentación complementaria, la autorregulación de la ingesta respetando las señales de hambre y saciedad, el consumo de frutas y verduras, evitar las bebidas azucaradas y mantener un estilo de vida activo son estrategias adecuadas para lograr un crecimiento saludable.

En la lucha contra la obesidad es un desafío para la Salud Pública instrumentar las medidas de apoyo nutricional a los grupos vulnerables en forma sostenida y con evaluación periódica de los resultados. Se necesita una combinación de estrategias como los impuestos a determinados alimentos ultraprocesados de alta densidad energética y baja calidad nutricional, los subsidios a frutas y verduras, la regulación de la publicidad, un rotulado de productos claro para el consumidor, los entornos escolares saludables y medidas para la promoción de la actividad física que, sumados a la educación alimentaria de la población, logren disminuir la magnitud de la obesidad en nuestro país.

Por último, y en el contexto de la situación actual, es preocupante el aumento y agravamiento de la obesidad infantil que se intuye desde el inicio de la pandemia COVID-19. Recientemente la Federación Latinoamericana de Sociedades de Obesidad (FLASO) publicó un documento sobre la relación entre ambas pandemias en Latinoamérica ${ }^{6}$. Allí se resalta que, durante el período de aislamiento, por temor al contagio al comprar productos frescos y el menor recurso económico, se incrementó el consumo de alimentos ultraprocesados de baja calidad nutricional. Una encuesta de percepción 
y actitudes de la población realizada por UNICEF en julio de 2020 a 2.525 hogares de 260 localidades (representativa de la totalidad de hogares urbanos), demostró que las restricciones económicas limitaron el consumo de alimentos variados y nutritivos ( $26 \%$ de los hogares tuvo que dejar de comprar algún alimento) e incrementaron la dependencia de los hogares a los apoyos alimentarios y al abastecimiento de los comedores escolares (10\% de los hogares) $)^{7}$. Este empeoramiento de la calidad de la alimentación junto con la inactividad física y el aumento de las horas de sedentarismo favorecen un balance energético positivo y el incremento de la obesidad. Este hecho, que los pediatras estamos percibiendo con la vuelta

\section{BIBLIOGRAFÍA}

1. ENNyS 2. Indicadores priorizados. Ministerio de Salud y Desarrollo Social, Septiembre 2019. Disponible en: https://cesni-biblioteca.org/2-encuesta-nacional-de-nutricion-y-salud-ennys-2-resumen-ejecutivo/.

2. UNICEF Argentina. Obesidad: una cuestión de derechos de niños, niñas y adolescentes. Disponible en: https:// www.unicef.org/argentina/informes/posicion-obesidad

3. Lin X, Yubin Lim I, WuY, et al. Developmental pathways to adiposity begin before birth and are influenced by genotype, prenatal environment and epigenome. BMC Medicine 2017; 15:50.

4. Whitaker R. Predicting preschooler obesity at birth: the role of maternal obesity in early pregnancy pediatrics 2004; 114:e29-e36. paulatina de los niños y adolescentes a la consulta, deberá documentarse a través de estudios epidemiológicos, así como su evolución pospandemia. Más allá que se resuelva la pandemia de COVID-19, los pediatras deberemos enfrentarnos con el crecimiento de la obesidad infantil, que sólo mejorará con un abordaje multidimensional.

\author{
Dra. Adriana Roussos \\ Médica Pediatra especialista en Nutrición \\ Hospital de Niños R. Gutiérrez, \\ Sección Nutrición y Diabetes
}

5. Farpour-Lambert N, Ells L, Martínez de Tejada B, et al. Obesity and weight gain in pregnancy and postpartum: an evidence review of lifestyle interventions to inform maternal and child health policies. Front Endocrinol 2018; 9:546.

6. UNICEF Argentina: El impacto de la pandemia COVID-19 en las familias con niños, niñas y adolescentes. Encuesta de percepción y actitudes de la población, segunda ola. Disponible en: https://www.unicef.org/argentina/ informes/encuesta-rapida-covid-19-informe-de-resultados-0.

7. Halpern B, da Costa Louzada ML, Aschner P, et al. Obesity and COVID-19 in Latin America: a tragedy of two pandemics. Official document of the Latin American Federation of Obesity Societies. Obesity Reviews 2020; 1-12. 